University of Wollongong

Research Online

Faculty of Engineering and Information

Faculty of Engineering and Information

Sciences - Papers: Part B

Sciences

2016

Biofouling Mitigation in Forward Osmosis Using Graphene Oxide Functionalized Thin-Film Composite Membranes

Francois Perreault

Yale University

Humberto Jaramillo

Yale University

Ming Xie

University of Wollongong, mx504@uowmail.edu.au

Mercy Ude

Yale University

Long D. Nghiem

University of Wollongong, longn@uow.edu.au

See next page for additional authors

Follow this and additional works at: https://ro.uow.edu.au/eispapers1

Part of the Engineering Commons, and the Science and Technology Studies Commons

Research Online is the open access institutional repository for the University of Wollongong. For further information contact the UOW Library: research-pubs@uow.edu.au 


\title{
Biofouling Mitigation in Forward Osmosis Using Graphene Oxide Functionalized Thin-Film Composite Membranes
}

\author{
Abstract \\ Forward osmosis (FO) is an emerging membrane process with potential applications in the treatment of \\ highly fouling feedwaters. However, biofouling, the adhesion of microorganisms to the membrane and the \\ subsequent formation of biofilms, remains a major limitation since antifouling membrane modifications \\ offer limited protection against biofouling. In this study, we evaluated the use of graphene oxide (GO) for \\ biofouling mitigation in FO. GO functionalization of thin-film composite membranes (GO-TFC) increased \\ the surface hydrophilicity and imparted antimicrobial activity to the membrane without altering its \\ transport properties. After $1 \mathrm{~h}$ of contact time, deposition and viability of Pseudomonas aeruginosa cells \\ on GO-TFC were reduced by $36 \%$ and $30 \%$, respectively, compared to pristine membranes. When GO-TFC \\ membranes were tested for treatment of an artificial secondary wastewater supplemented with P. \\ aeruginosa, membrane biofouling was reduced by $50 \%$ after $24 \mathrm{~h}$ of operation. This biofouling resistance \\ is attributed to the reduced accumulation of microbial biomass on GO-TFC compared to pristine \\ membranes. In addition, confocal microscopy demonstrated that cells deposited on the membrane \\ surface are inactivated, resulting in a layer of dead cells on GO-TFC that limit biofilm formation. These \\ findings highlight the potential of GO to be used for biofouling mitigation in FO.

\section{Disciplines} \\ Engineering | Science and Technology Studies

\section{Publication Details} \\ Perreault, F., Jaramillo, H., Xie, M., Ude, M., Nghiem, L. D. \& Elimelech, M. (2016). Biofouling Mitigation in \\ Forward Osmosis Using Graphene Oxide Functionalized Thin-Film Composite Membranes. Environmental \\ Science and Technology (Washington), 50 (11), 5840-5848.
}

\section{Authors}

Francois Perreault, Humberto Jaramillo, Ming Xie, Mercy Ude, Long D. Nghiem, and Menachem Elimelech 


\section{Biofouling Mitigation in Forward Osmosis using Graphene Oxide Functionalized Thin-Film Composite Membranes}

\author{
Environmental Science \& Technology
}

Revised: April 2016

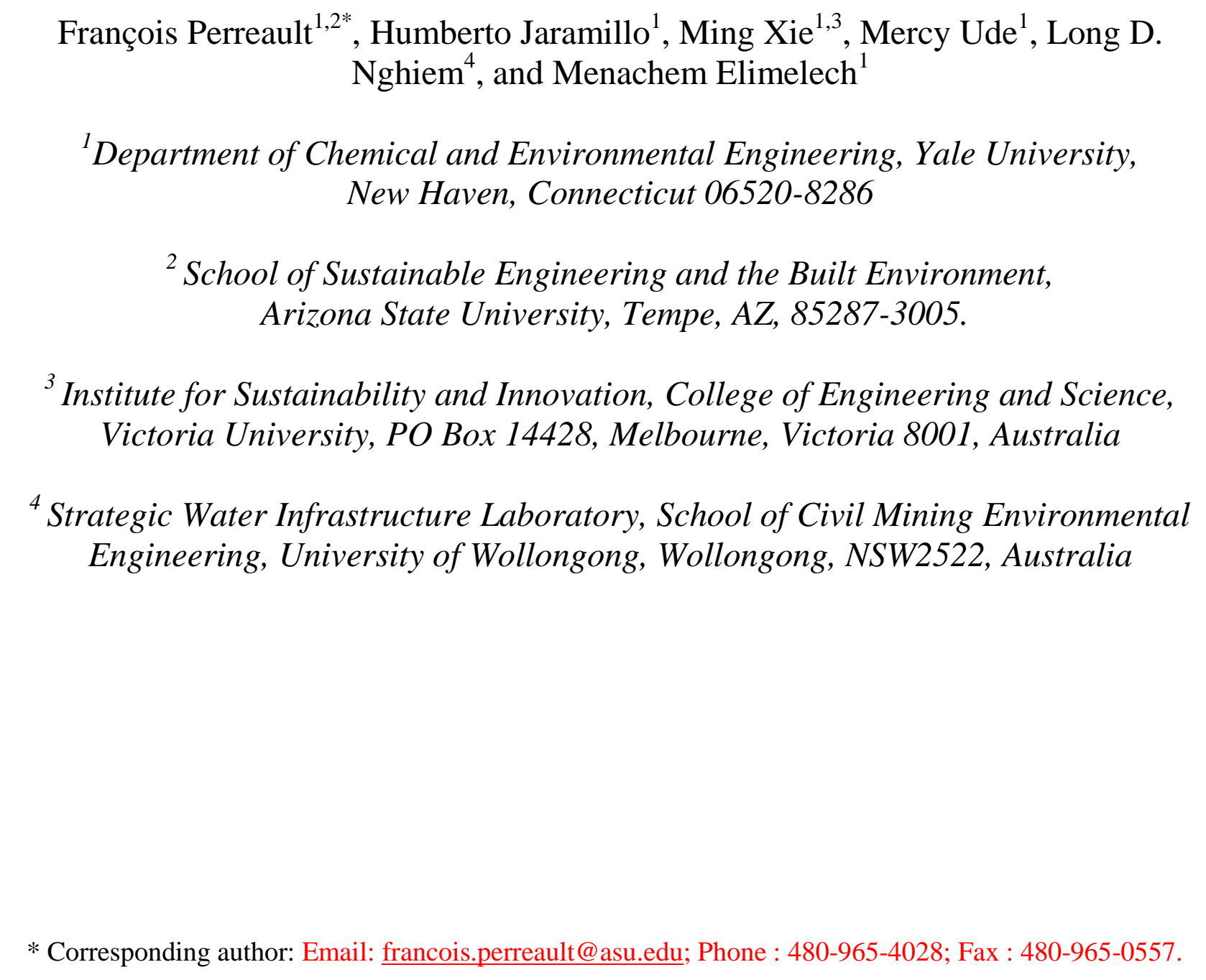

${ }^{3}$ Institute for Sustainability and Innovation, College of Engineering and Science, Victoria University, PO Box 14428, Melbourne, Victoria 8001, Australia

${ }^{4}$ Strategic Water Infrastructure Laboratory, School of Civil Mining Environmental Engineering, University of Wollongong, Wollongong, NSW2522, Australia

* Corresponding author: Email: francois.perreault@asu.edu; Phone : 480-965-4028; Fax : 480-965-0557. 


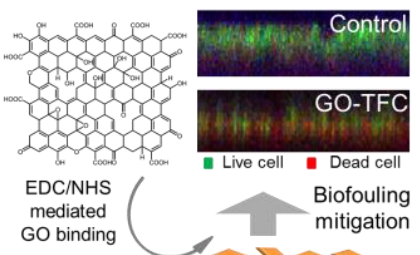

42

43

44 


\section{ABSTRACT}

46 Forward osmosis (FO) is an emerging membrane process with potential applications in the

47 treatment of highly fouling feedwaters. However, biofouling, the adhesion of microorganisms to 48 the membrane and the subsequent formation of biofilms, remains a major limitation since 49 antifouling membrane modifications offer limited protection against biofouling. In this study, we 50 evaluated the use of graphene oxide (GO) for biofouling mitigation in FO. GO functionalization

51 of thin-film composite membranes (GO-TFC) increased the surface hydrophilicity and imparted 52 antimicrobial activity to the membrane without scarifying its transport properties. After $1 \mathrm{~h}$ of 53 contact time, deposition and viability of Pseudomonas aeruginosa cells on GO-TFC were 54 reduced by $36 \%$ and $30 \%$, respectively, compared to pristine membranes. When GO-TFC 55 membranes were tested for treatment of an artificial secondary wastewater supplemented with $P$. 56 aeruginosa, membrane biofouling was reduced by $50 \%$ after 24 hours of operation. This 57 biofouling resistance is attributed to the reduced accumulation of microbial biomass on GO-TFC 58 compared to pristine membranes. In addition, confocal microscopy demonstrated that cells 59 deposited on the membrane surface are inactivated, resulting in a layer of dead cells on GO-TFC 60 that limit biofilm formation. These findings highlight the potential of GO to be used for 61 biofouling mitigation in FO membrane design. 
64 With a growing world population, global climate change, and intensification of human activities, 65 water availability is becoming one of the most important environmental challenges facing 66 humanity. ${ }^{1}$ Membrane-based technologies for water treatment, water reclamation, and 67 desalination are some of the most effective strategies to address global water quality and scarcity 68 issues. $^{1-3}$ However, membranes are prone to fouling, that is, the accumulation of organic, 69 inorganic, or biological foulants on the membrane, which decrease permeate flux, membrane 70 selectivity, and useful lifetime. ${ }^{1}$ The design of effective fouling control strategies is therefore one 71 of the main technical challenges in membrane-based water treatment.

72 Forward osmosis (FO) is an emerging membrane process that uses the osmotic difference 73 between a concentrated draw solution and a dilute feed solution to induce spontaneous solvent 74 permeation through a semipermeable membrane. ${ }^{4}$ This osmotic driving force results in a foulant 75 layer that is less compact and more easily cleanable than in pressure-driven processes such as 76 reverse osmosis (RO). ${ }^{5,6}$ As a result, FO has emerged as a practical approach to treat waters with 77 high fouling potential like wastewater or activated sludge. ${ }^{4,7,8}$ However, fouling is still 78 detrimental to FO operations due to cake-enhanced concentration polarization, which decreases 79 the osmotic driving force for permeation and demands frequent system interruptions for 80 membrane cleaning. . ${ }^{4,7}$ Therefore, improving the resistance of membranes to fouling can 81 contribute to the successful implementation of FO technologies.

82 FO membrane fouling propensity is associated with the membrane surface roughness, 83 relative hydrophobicity, and its high density of carboxyl groups. ${ }^{9,10}$ These factors are typically

84 found in the polyamide thin-film composite (TFC) membranes used in FO. To avoid excessive 85 fouling, modified TFC membranes have been developed to decrease foulant adsorption. ${ }^{10}$ 86 Common antifouling modifications include polymer brushes, zwitterions, and superhydrophilic 87 nanomaterials. ${ }^{10-13}$ Such modifications were shown to improve the membrane resistance to 88 fouling caused by organic molecules like proteins, polysaccharides, or natural organic matter. ${ }^{11-}$ 89

91 foulants. In FO-based treatment of secondary wastewater, fouling was found to be dominated by

92 biopolymers, proteins, and microorganisms. ${ }^{14}$ Biological fouling, or biofouling, involves the 
93 adsorption of microorganisms to the membrane and their development into microbial 94 communities enclosed in extracellular polymeric substances (EPS). ${ }^{15}$ The contribution of 95 biofouling makes fouling mitigation more challenging since membrane modifications aiming to 96 reduce foulant adsorption often have a limited effect on biofilm formation. ${ }^{16,17}$

97 To specifically target biofouling, antimicrobial properties have been imparted to 98 membranes. ${ }^{18-20}$ Antimicrobial membranesinactivate bacterial cells at contact, reducing the 99 initial rate of biofilm formation. ${ }^{20}$ However, their long-term efficiency is limited by the eventual 100 depletion of biocide or the accumulation of dead cells on the surface, which will shield the 101 antimicrobial material. Recent efforts have thus been made to design membranes with both 102 antimicrobial and antifouling properties, where membranes are modified in multiple steps with 103 sequential grafting of polymer brushes or zwitterions, for antifouling properties, and 104 nanoparticles or polycations, for antimicrobial activity. ${ }^{21-23}$ While these modifications represent 105 more complex membrane functionalization, the combination of antifouling and antimicrobial 106 properties was highlighted as the most effective approach to mitigate membrane biofouling. ${ }^{20}$

107 Graphene oxide (GO) is a carbon-based nanomaterial composed of a single layer of $s p^{2}$ 108 bonded carbon decorated with a high density of oxygen functional groups. ${ }^{24}$ Due to its high 109 surface area and colloidal stability in aqueous conditions, GO is extensively investigated as a 110 platform material for novel membrane designs. ${ }^{25,26}$ Notably, its incorporation into membranes 111 was found to improve their resistance to fouling by reducing both surface roughness and 112 hydrophobicity. ${ }^{27,28} \mathrm{GO}$ also possesses bactericidal properties and can induce a disruption of the 113 cell membrane when bacteria come into contact with GO. ${ }^{26,29-31}$ Therefore, GO may be an 114 excellent material for the development of biofilm-resistant membranes as it can impart both 115 antimicrobial and antifouling properties to a surface. Membrane surface functionalization with 116 GO was previously shown to impart antimicrobial properties to its active layer; ${ }^{30,32}$ however, its 117 biofouling mitigation potential remains to be demonstrated in membrane operations.

118 In this paper, we evaluated the use of $\mathrm{GO}$ for biofouling mitigation in FO. GO119 functionalized membranes were exposed to an artificial secondary wastewater feed, to which the 120 biofilm-forming bacterium Pseudomonas aeruginosa was added, and tested in a bench-scale 121 cross-flow FO unit. We demonstrated that when membranes are functionalized with GO (GO122 TFC), water flux decline due to biofouling is reduced. Analysis of the structure and composition 
123 of the biofilm formed on the membrane, in conjunction with a characterization of the change in 124 surface properties imparted by GO, provided insights on the mechanisms involved in biofouling 125 mitigation by GO. These findings highlight the potential of GO to be utilized as a biofouling 126 control material in FO membrane design.

\section{MATERIALS AND METHODS}

Graphene Oxide Synthesis and Characterization. GO was produced by chemical oxidation of graphite by $\mathrm{KMnO}_{4}$ in a mixture of $\mathrm{H}_{2} \mathrm{SO}_{4}$ and $\mathrm{H}_{3} \mathrm{PO}_{4}$, as previously described. ${ }^{33}$

131 Spectroscopic characterization was realized on dry GO powders. Raman spectroscopy was 132 performed on a Horiba Jobin Yvon HR-800 spectrometer with a $532 \mathrm{~nm}$ excitation. Fourier133 Transformed Infrared (FTIR) spectra were collected using a Thermo Nicolet 6700 spectrometer. 134 X-ray photoelectron spectroscopy (XPS) was performed on a ThermoScientific ESCALAB 250 135 with a monochromatized AI X-ray source. For microscopy analysis, GO sheets were drop-casted 136 on a silicon wafer. Atomic Force Microscopy analysis was performed in tapping mode with a 137 Bruker Multimode AFM (Digital Instruments, Plainview, NY) equipped with a Tap300Al-G 138 cantilever (BudgetSensors, Sofia, Bulgaria). SEM analyses were done on a Hitachi SU-70 microscope (Hitachi High Technologies America, Inc., Clarksburg, MD). The antimicrobial activity of GO was verified by measuring the cell viability of $P$. aeruginosa cells deposited on a 141 pure GO layer. Cell viability was measured after $1 \mathrm{~h}$ by staining the cells with SYTO 9 and 142 propidium iodide (PI) and quantifying live and dead cells with an Axiovert 200M 143 epifluorescence microscope (Carl Zeiss Inc., Thornwood, NY). Further information on GO 144 synthesis and characterization is given in the Supporting Information (SI).

145 Membrane Functionalization. GO was covalently bound to FO membranes by a 146 previously described amide coupling reaction. ${ }^{32}$ Briefly, the carboxyl groups on the membrane 147 polyamide layer are converted to amine-reactive esters by reaction with $4 \mathrm{mM} \mathrm{N}-(3-$ 148 dimethylaminopropyl)-N'-ethylcarbodiimide hydrochloride (EDC) and 10 mM N149 hydroxysuccinimide (NHS) for $1 \mathrm{~h}$. The amine-reactive esters are then used to attach 150 ethylenediamine to the membrane. Finally, GO $(10 \mathrm{mg})$ is reacted with $2 \mathrm{mM}$ EDC and $5 \mathrm{mM}$ 151 NHS for $15 \mathrm{~min}$, to activate its carboxyl groups, and placed in contact with the ethylenediamine152 rich membrane for amide coupling. The detailed functionalization protocol is provided in the SI. 

800 spectrometer using a $532 \mathrm{~nm}$ laser excitation. For SEM imaging, samples were sputtercoated with chromium and imaged with a Hitachi SU-70 microscope. Membrane hydrophilicity was evaluated by the sessile drop method using a Theta Lite Optical Tensiometer TL100 (Attension, Espoo, Finland), using a drop volume of $5 \mu \mathrm{L}$. Surface roughness was measured in tapping mode with a Dimension Icon AFM equipped with a SNL-10 SiN cantilever (Bruker, Santa Barbara, CA). The membrane water permeability, $A$, salt permeability, $B$, and structural parameter, $S$, were determined according to a method previously described. ${ }^{34}$ Draw solution concentrations of approximately $0.2,0.4,0.7$, and $1.2 \mathrm{M} \mathrm{NaCl}$, and $\mathrm{DI}$ as feed solution, were used for the different characterization steps.

Bacterial Adhesion and Viability. Membrane coupons of $3.5 \mathrm{~cm}^{2}$ were placed in plastic holders leaving only the active layer exposed. A $3 \mathrm{~mL}$ suspension volume of $P$. aeruginosa $\left(\sim 10^{8}\right.$ $\mathrm{CFU} \mathrm{mL} \mathrm{m}^{-1}$ ) was contacted with the surface for $1 \mathrm{~h}$ at room temperature. The membranes were washed to remove non-attached cells and cell viability was determined by staining the cells with $3.34 \mu \mathrm{M}$ SYTO 9 and $20 \mu \mathrm{M}$ PI. The membranes were rinsed twice before mounting on a microscopic slide. Ten pictures per replicate were taken with an Axiovert 200M epifluorescence microscope (Carl Zeiss Inc., Thornwood, NY) and analyzed with Image J (National Institutes of Health, MD).

AFM Adhesion Force Measurements. Adhesion forces between the membrane and a 4 $172 \mu \mathrm{m}$ carboxylated latex particle (Life Technologies, Eugene, OR) were measured on a Dimension 173 Icon AFM (Bruker, Santa Barbara, CA). Particle-functionalized AFM probes were prepared 174 according to a procedure previously described. ${ }^{35}$ Force measurements were collected in synthetic 175 wastewater media using a trigger force of $1 \mathrm{nN}$, a ramp size of $1 \mu \mathrm{m}$, and a ramp rate of $0.5 \mathrm{~Hz}$. More details on AFM measurements are given in the SI.

Membrane Biofouling Experiments. Biofouling experiments were carried out in a 178 closed-loop, bench-scale FO unit. The active membrane area was $20.0 \mathrm{~cm}^{2}$. An artificial 179 secondary wastewater medium (ionic strength of $16 \mathrm{mM}, \mathrm{pH}$ of 7.6) was used as a feed solution 180 (detailed in Table S1). ${ }^{36}$ The draw solution was prepared using $\mathrm{NaCl}$ and the concentration 181 adjusted to achieve an initial water flux of $20 \pm 1 \mathrm{~L} \mathrm{~m}^{-2} \mathrm{~h}^{-1}(\sim 1 \mathrm{M} \mathrm{NaCl} \mathrm{draw})$. For each 182 membrane, a baseline run was conducted without bacteria to account for the dilution of the draw 
183 solution during experiments. The permeate flux was stabilized at $20 \pm 1 \mathrm{~L} \mathrm{~m}^{-2} \mathrm{~h}^{-1}$ before addition 184 of $P$. aeruginosa to an initial concentration of $\sim 6.0 \times 10^{7} \mathrm{CFU} \mathrm{L}^{-1}$. The FO system was operated 185 for 24 hours at a flow rate of $8.5 \mathrm{~cm} \mathrm{~s}^{-1}$. The permeate flux was continuously monitored and the 186 temperature maintained at $25 \pm 1{ }^{\circ} \mathrm{C}$. At the end of the biofouling experiment, membrane 187 coupons were cut for biofilm characterization. Biofouling experimental procedures are further detailed in the SI.

Biofilm Characterization. Membrane coupons $\left(1 \mathrm{~cm}^{2}\right)$ were cut from the center of the biofouled membrane, stained with SYTO 9, PI, and concavalin A (Con A), and mounted in a custom-made chamber for confocal laser scanning microscopy (CLSM). ${ }^{37}$ CLSM images were 192 captured using a Zeiss LSM 510 (Carl Zeiss, Inc., Thornwood, NY) equipped with a Plan193 Apochromat 20×/0.8 numerical aperture objective. Image analysis was performed using Auto194 PHLIP-ML, ImageJ, and MATLAB. Biovolumes were determined for the live cells, dead cells, 195 and EPS (con A-stained) components of the biofilm. Total biovolume and thickness were 196 calculated by summing live cells, dead cells, and EPS. Quantitative analysis of the biofilm was also performed by measuring the total protein and organic carbon (TOC) extracted from the membrane surface. Complete biofilm characterization procedures are detailed in the SI.

\section{RESULTS AND DISCUSSION}

201 Chemical Oxidation of Graphite to Graphene Oxide. GO was produced by chemical 202 oxidation of graphite by $\mathrm{KMnO}_{4}$ in concentrated sulfuric and phosphoric acid. ${ }^{33}$ These oxidative conditions generated multiple defect sites in the graphitic structure, as indicated by the higher D band intensity in the Raman spectrum of GO (Figure 1a). In carbon nanomaterials, the G band originates from the $s p^{2}$-bonded carbon structure while the D band reflects the disorder in the $s p^{2}$ structure caused by the presence of defects and sheet edges. ${ }^{24,38}$ Compared to graphite, the G/D

208 nature of those defects was identified by FTIR and XPS spectroscopy. The FTIR spectrum shows 209 characteristic peaks for $s^{2} \mathrm{C}=\mathrm{C}$ bonds $\left(1615 \mathrm{~cm}^{-1}\right)$ as well as oxygenated $\mathrm{C}-\mathrm{O}\left(1220 \mathrm{~cm}^{-1}\right)$, $210 \mathrm{C}=\mathrm{O}\left(1720 \mathrm{~cm}^{-1}\right)$, and $\mathrm{O}-\mathrm{H}\left(3400-3600 \mathrm{~cm}^{-1}\right)$ groups (Figure 1b). Analysis of the XPS C1s 211 spectra reveals that the main oxygenated functional groups are $\mathrm{C}-\mathrm{O}(52 \%), \mathrm{C}=\mathrm{O}(7.1 \%)$ and $\mathrm{O}-$ $212 \mathrm{C}=\mathrm{O}(5 \%)$ (Figure 1c). 
The presence of oxygen functional groups in the graphitic structure increases the 214 interlamellar spacing in graphite and allows water to seep in between the graphene layers, 215 facilitating their exfoliation by ultrasonication. ${ }^{24,26}$ AFM topographical analysis showed that the 216 exfoliated sheets were $\sim 1.4 \mathrm{~nm}$ in thickness (Figure $1 \mathrm{~d}$, e), which is equivalent to single layer 217 GO sheets. ${ }^{39}$ The average sheet dimension, determined by SEM imaging, was found to be 0.19 $218 \mu \mathrm{m}^{2}$. A representative SEM image of GO sheets deposited on a silicon wafer is presented in $219 \quad$ Figure S1.

Graphene Oxide Sheets Possess Strong Antimicrobial Properties. The antimicrobial properties of GO were demonstrated for a wide variety of microorganisms. ${ }^{26,29-31,40}$ Cell 222 inactivation has been proposed to be mediated by physical and oxidative interactions leading to a 223 disruption of the membrane integrity and to cell death. ${ }^{26,29,32,41,42}$ However, the antimicrobial 224 potential can differ significantly between different GO materials, with some studies indicating 225 high bacterial inactivation while others report no observable toxicity. ${ }^{29,32,43}$ This discrepancy can 226 be due to the heterogeneous nature of GO materials generated by different oxidation 227 procedures. $^{44}$

Considering this variable nature of GO, the antimicrobial potential of the GO material produced by our chemical oxidation procedure was verified. When $P$. aeruginosa cells are 230 exposed to a pure GO layer formed by vacuum filtration on a polycarbonate membrane, a 231 decrease in cell viability is observed (Figure S2 a, b). After $1 \mathrm{~h}$ of exposure, cell viability 232 decreases from $82 \%$ on the control polycarbonate filter to $20 \%$ on GO (Figure S2c). Previous 233 studies on the antimicrobial activity of GO deposited on a surface report bacterial inactivation 234 ranging from 59 to $89 \%$ for exposure time of $1-3 \mathrm{~h}$ with E. coli. ${ }^{29,45,46}$ Therefore, the GO 235 produced in this study possesses high antimicrobial activity.

Graphene Oxide Functionalization Changes Surface Properties without Altering

237 Transport Properties. GO sheets were grafted to the polyamide layer through a covalent amide 238 bond formation using ethylenediamine as a cross-linker, as previously described. ${ }^{32}$ Successful 239 binding of GO was indicated by SEM imaging. Compared to the pristine membrane (Figure 2a), 240 GO can be visualized as a sheet-like material covering the active layer of the membrane (Figure

241 2b). This material was confirmed to be GO by Raman spectroscopy, using the $\mathrm{I}_{1147} / \mathrm{I}_{1585}$ ratio. ${ }^{32}$ 242 In TFC membranes, the two dominant Raman peaks originate from the symmetric $\mathrm{C}-\mathrm{O}-\mathrm{C}$ 
243 stretching and phenyl ring vibration of polysulfone, at 1147 and $1585 \mathrm{~cm}^{-1}$ (Figure 2c). ${ }^{47,48} \mathrm{GO}$, 244 when bound to the membrane, contributes to the Raman signal at $1585 \mathrm{~cm}^{-1}$ due to its $\mathrm{G}$ band,

245 while its Raman signal at $1147 \mathrm{~cm}^{-1}$ is minimal (Figure 1a). After functionalization with GO, the $246 \mathrm{I}_{1147} / \mathrm{I}_{1585}$ decreases from $1.47 \pm 0.02$ for Ctrl membranes to $1.22 \pm 0.09$ for GO-TFC membranes 247 (Student $t$-test, $p<0.05$ ), confirming the attachment of GO (Figure 2c). Considering the surface 248 chemistry and covalent binding reaction used for GO surface modification, the amount of GO 249 covering the membrane is hypothesized to be mostly a monolayer of GO, with some overlapping 250 between neighboring GO sheets.

251 When Ctrl and GO-TFC membranes are characterized using the four-step FO 252 characterization protocol established by Tiraferri et al., ${ }^{34}$ no significant impact of GO 253 functionalization is observed on the transport properties of the membrane (Figure S3). These 254 results are in agreement with previous findings showing that the addition of multiple GO layers 255 on TFC membranes did not reduce the water permeability of the membrane. ${ }^{28}$ However, the 256 presence of GO on the active layer changes the surface properties of the membrane. After 257 functionalization with $\mathrm{GO}$, the water contact angle of the membrane decreases from $35 \pm 4^{\circ}$ to $25825 \pm 3^{\circ}$, indicating that the surface is rendered more hydrophilic (Figure 3a). This change in 259 hydrophilicity cannot be attributed to a change in surface roughness since AFM analysis of 260 pristine and GO-TFC membranes reveals no change in the surface roughness after GO 261 functionalization (Figure S4). Both membranes have an average surface roughness $\left(r_{m s}\right)$ of $\sim 70$ $262 \mathrm{~nm}$ (Figure 3b). Therefore, the increased surface hydrophilicity can be attributed to the high 263 density of oxygen functional groups in $\mathrm{GO}^{24}$

Graphene Oxide Imparts Anti-adhesive and Antimicrobial Surface Properties. By 265 increasing surface hydrophilicity, foulant adhesion can be decreased. ${ }^{10,20}$ This anti-adhesive 266 effect is due to the formation of a hydration layer opposing the adsorption of biomolecules to the 267 surface. ${ }^{49}$ Given this role of hydrophilicity in fouling, increasing the hydrophilicity of the 268 membranes is often used as a strategy to improve their fouling resistance. ${ }^{10-12,50}$

269 The anti-adhesive properties of GO-TFC were verified by chemical force microscopy 270 using a carboxylated latex particle attached to a tipless AFM cantilever (Figure S5). ${ }^{35}$ The high 271 density of carboxyl groups on the particle allows this colloidal probe to be used as a model for 272 fouling since carboxylic groups play an important role in the calcium-mediated foulant 
273 complexation to membranes. ${ }^{51,52}$ Chemical force spectroscopy reveals that GO imparts anti274 adhesive properties to the surface. Compared to a Ctrl membrane, where the average adhesion 275 force between the colloidal probe and the membrane is $-0.49 \mathrm{mN} \mathrm{m}^{-1}$ (Figure 4a), GO-TFC 276 membranes have an average adhesion force of $-0.15 \mathrm{mN} \mathrm{m}^{-1}$ (Figure $4 \mathrm{~b}$ ). The adhesion force 277 distribution on GO-TFC is also characterized with a higher frequency of "NO" events, where the 278 interaction between the probe and the membrane is repulsive and no adhesion is measured. In 279 GO-TFC membranes, $55 \%$ of the measurements showed no adhesion, compared to $27 \%$ for Ctrl 280 membranes (Figure 4a, b).

281 Reduced protein adsorption was previously shown for different types of GO-blended 282 polymeric membranes. ${ }^{27,53,54}$ Similarly, surface-functionalized RO TFC membranes, where GO 283 was assembled on the surface via a layer-by-layer approach, also showed a reduced adsorption of 284 proteins. ${ }^{28}$ Lower fouling propensity of GO-functionalized surfaces can be attributed to an 285 increase in surface hydrophilicity and a smoothing of the membrane surface. ${ }^{28}$ However, for GO286 TFC, no change in surface roughness is observed after functionalization with GO, suggesting 287 that surface hydrophilicity was the main reason for its anti-adhesive properties. Increased 288 hydrophilicity was also proposed as the mechanism for the lower fouling propensity of 289 poly(vinylidene fluoride) and polyethersulfone membranes mixed with GO. ${ }^{53,54}$

290 The anti-adhesive properties of GO-TFC membranes were further confirmed by 291 evaluating bacterial adhesion to the membrane. After a 1-h contact time of a $P$. aeruginosa 292 suspension to Ctrl or GO-TFC membranes, cells attached to the membrane were stained with 293 SYTO 9 and PI, enabling cell enumeration and viability assessment (Figure 4c). A lower amount 294 of bacteria is found attached to GO-TFC compared to Ctrl membranes. The number of bacterial 295 cells decreases from $50 \times 10^{6}$ cells per $\mathrm{cm}^{2}$ to $32 \times 10^{6}$ cells per $\mathrm{cm}^{2}$ for Ctrl and GO-TFC 296 membranes, respectively (Figure 4d). At the same time, cell viability of bacteria on the surface is 297 also affected, decreasing from $92 \%$ for cells attached to the Ctrl membrane to $62 \%$ for GO-TFC 298 membrane (Figure 4d). Therefore, GO sheets are still active when bound to the membrane and 299 impart antimicrobial properties as well as anti-adhesive properties to the membrane.

300 Graphene Oxide Mitigates Biofouling in Forward Osmosis. The anti-adhesive and 301 antimicrobial properties imparted by GO suggest promising biofouling resistance in GO-TFC 302 membranes. However, it should be noted that short-term static assays are not always indicative 
303 of biofouling resistance in membranes. For ultrafiltration and nanofiltration membranes modified 304 with polydopamine or polydopamine-g-poly(ethylene glycol), biofouling was not affected 305 despite both reduced protein adsorption and P. aeruginosa bacterial adhesion in short term static 306 assays. ${ }^{17}$ A similar outcome was obtained with TFC RO membranes modified with anti-adhesive 307 polymer brushes. $^{16}$

In order to accurately determine the biofouling mitigation potential of GO-TFC membranes, dynamic biofouling assays were conducted in a lab-scale cross-flow FO unit. An 310 artificial secondary wastewater medium was used as a feed solution and P. aeruginosa were 311 added at an initial concentration of $\sim 6.0 \times 10^{7} \mathrm{CFU} \mathrm{L}^{-1}$. Over the course of $24 \mathrm{~h}$, a gradual 312 decline was observed in the permeate flux due to the formation of a biofilm on the membrane. 313 For Ctrl membranes, the flux decline due to biofouling reaches $40 \%$ of the initial flux after $24 \mathrm{~h}$ 314 of operation, while flux decline for the GO-TFC membranes was 20\% (Figure 5a). GO 315 functionalization was thus able to reduce the effect of biofouling on membrane performance.

To understand the role of GO in biofouling mitigation, the membrane was removed from the cell after the $24 \mathrm{~h}$ of filtration, and stained for CLSM analysis. Analysis of the side-view of the biofilm reveals important structural differences between the biofilms formed on Ctrl and GOTFC membranes (Figure 5b). The biofilm layer on GO-TFC is thinner than on Ctrl membranes and a layer of dead cells, shown in red by PI staining, can be observed in the bottom part of the biofilm in contact with the GO-functionalized surface (Figure 5b). This layer of dead cells 322 cannot be observed on the Ctrl membrane, indicating that the antimicrobial activity provided by 323 GO is inactivating bacterial cells in contact with the functionalized surface.

Analysis of the CLSM images was used to quantify the biovolumes of live cells, dead 325 cells, and EPS in the biofilm. These results show that biofilm formed after 24 hours on GO-TFC 326 membranes is thinner and composed of fewer live cells, more dead cells, and smaller EPS 327 biovolumes than biofilms formed on Ctrl membranes (Table 1). Quantitative analysis of the 328 biomass accumulated on the membrane confirms these findings; GO-TFC membranes have less 329 total protein and TOC, both related to bacterial biomass per membrane area than Ctrl membranes 330 (Table 1). Altogether, these results indicate a sparser biofilm development on GO-TFC 331 membranes, an observation that is in agreement with CLSM images (Figure 5c, d). Reduced 
332 accumulation and growth of biomass on the membrane is likely contributed to the lower flux

333 decline observed for GO-TFC membranes under dynamic biofouling conditions.

Implications for Graphene Oxide-Based Biofilm Control. Although numerous studies reported anti-adhesive or antimicrobial membranes using GO, ${ }^{27,30,32,53-55}$ very few up to now 336 addressed the more complex issue of biofouling. Biofilm mitigation by GO has been demonstrated for model surfaces like indium tin oxide, ${ }^{56}$ or in ultrafiltration membranes used for

338 membrane bioreactors. ${ }^{57}$ However, for ultrafiltration membranes, biofouling mitigation was 339 entirely attributed to the anti-adhesive properties of GO incorporated in the polysulfone matrix. ${ }^{57}$ 340 In our study, our results show that bacterial inactivation induced by GO sheets also contribute to 341 the reduced biofilm formation on GO-functionalized TFC membranes. These findings provide 342 useful insights into the design of GO-based surfaces for biofouling control, where both anti343 adhesive and antimicrobial properties must be considered. The simplicity of membrane 344 functionalization with GO, the absence of detrimental effects on the membrane transport 345 properties, and the possibility of improving both the antimicrobial activity and the hydrophilicity 346 of the membrane selective layer, through changes in sheet size, ${ }^{46}$ oxidation level, ${ }^{29}$ and nanoscale 347 topography, ${ }^{58}$ render GO a viable and attractive material for anti-biofouling membrane 348 development. Future investigations should focus on fine-tuning the physicochemical 349 characteristics of GO to improve both these functionalities. Long-term studies are also needed to 350 assess the stability of the antimicrobial and antifouling properties of GO when exposed to 351 complex water chemistries.

\section{ACKNOWLEDGEMENT}

355 F.P. acknowledges the financial support from the Natural Sciences and Engineering Research 356 Council of Canada. H.J. acknowledges the support of the US National Science Foundation 357 Graduate Research Fellowship (2013162783). Facilities used were supported by the Yale 358 Institute of Nanoscale and Quantum Engineering and NSF MRSEC DMR 1119826. We also 359 thank Prof. Kanani Lee for granting access to the Raman spectrometer. 
362 Additional Material and Methods; SEM micrograph of GO sheets deposited on a silicon wafer 363 (Figure S1); Antimicrobial activity of the produced GO sheets (Figure S2); Membrane transport 364 properties of Ctrl and GO-functionalized TFC membranes (Figure S3);AFM 3-D topographical 365 image of Ctrl and GO-TFC membranes (Figure S4); SEM micrograph of the carboxylated366 particle attached on a tipless silicon nitride cantilever. (Figure S5); Zeta potential of Ctrl and 367 GO-TFC membranes (Figure S6); Synthetic wastewater composition (Table S1). This material is 368 available free of charge via the Internet at http://pubs.acs.org. 
(1) Shannon, M. A.; Bohn, P. W.; Elimelech, M.; Georgiadis, J. G.; Mariñas, B. J.; Mayes, A. M.; Marinas, B. J.; Mayes, A. M.; Mariñas, B. J.; Mayes, A. M. Science and technology

(2) Wintgens, T.; Melin, T.; Schäfer, A.; Khan, S.; Muston, M.; Bixio, D.; Thoeye, C. The role of membrane processes in municipal wastewater reclamation and reuse. Desalination 2005, 178, 1-11.

(3) Elimelech, M.; Phillip, W. A. The future of seawater desalination: energy, technology, and the environment. Science 2011, 333 (6043), 712-717.

(4) Shaffer, D. L.; Werber, J. R.; Jaramillo, H.; Lin, S.; Elimelech, M. Forward osmosis : Where are we now? Desalination 2015, 356, 271-284.

(5) Mi, B.; Elimelech, M. Organic fouling of forward osmosis membranes: Fouling reversibility and cleaning without chemical reagents. J. Memb. Sci. 2010, 348, 337-345.

(6) Lee, S.; Boo, C.; Elimelech, M.; Hong, S. Comparison of fouling behavior in forward osmosis (FO) and reverse osmosis (RO). J. Memb. Sci. 2010, 365, 34-39.

(7) Lutchmiah, K.; Verliefde, R. D.; Roest, K.; Rietveld, L. C.; Cornelissen, E. R. Forward osmosis for application in wastewater treatment: a review. Water Res. 2014, 58, 179-197.

(8) Achilli, A.; Cath, T. Y.; Marchand, E. A.; Childress, A. E. The forward osmosis membrane bioreactor: A low fouling alternative to MBR processes. Desalination 2009, $238,10-21$.

(9) Mo, Y.; Tiraferri, A.; Yip, N. Y.; Adout, A.; Huang, X.; Elimelech, M. Improved antifouling properties of polyamide nanofiltration membranes by reducing the density of surface carboxyl groups. Environ. Sci. Technol. 2012, 46, 13253-13261.

(10) Rana, D.; Matsuura, T. Surface modifications for antifouling membranes. Chem. Rev. 2010, 110, 2448-2471.

(11) Romero-Vargas Castrillón, S.; Lu, X.; Shaffer, D. L.; Elimelech, M. Amine enrichment and poly(ethylene glycol) (PEG) surface modification of thin-film composite forward osmosis membranes for organic fouling control. J. Memb. Sci. 2014, 450, 331-339.

(12) Tiraferri, A.; Kang, Y.; Giannelis, E. P.; Elimelech, M. Superhydrophilic thin-film composite forward osmosis membranes for organic fouling control: Fouling behavior and antifouling mechanisms. Environ. Sci. Technol. 2012, 46, 11135-11144.

(13) Yu, H. Y.; Kang, Y.; Liu, Y.; Mi, B. Grafting polyzwitterions onto polyamide by click chemistry and nucleophilic substitution on nitrogen: A novel approach to enhance membrane fouling resistance. J. Memb. Sci. 2014, 449, 50-57.

(14) Valladares Linares, R.; Yangali-Quintanilla, V.; Li, Z.; Amy, G. NOM and TEP fouling of a forward osmosis (FO) membrane: Foulant identification and cleaning. J. Memb. Sci. 2012, 421-422, 217-224. 
408

(15) Flemming, H. C.; Schaule, G.; Griebe, T.; Schmitt, J.; Tamachkiarowa, a. Biofouling the Achilles heel of membrane processes. Desalination 1997, 113, 215-225.

(16) Bernstein, R.; Freger, V.; Lee, J.-H.; Kim, Y.-G.; Lee, J.; Herzberg, M. "Should I stay or should I go?" Bacterial attachment vs biofilm formation on surface-modified membranes. Biofouling 2014, 30, 367-376.

(17) Miller, D. J.; Araújo, P. A.; Correia, P. B.; Ramsey, M. M.; Kruithof, J. C.; van Loosdrecht, M. C. M.; Freeman, B. D.; Paul, D. R.; Whiteley, M.; Vrouwenvelder, J. S. Short-term adhesion and long-term biofouling testing of polydopamine and poly(ethylene glycol) surface modifications of membranes and feed spacers for biofouling control. Water Res. 2012, 46, 3737-3753.

(18) Blok, A. J.; Chhasatia, R.; Dilag, J.; Ellis, A. V. Surface initiated polydopamine grafted poly([2-(methacryoyloxy)ethyl] trimethylammonium chloride) coatings to produce reverse osmosis desalination membranes with anti-biofouling properties. J. Memb. Sci. 2014, 468, 216-223.

(19) Ben-Sasson, M.; Lu, X.; Bar-Zeev, E.; Zodrow, K. R.; Nejati, S.; Qi, G.; Giannelis, E. P.; Elimelech, M. In situ formation of silver nanoparticles on thin-film composite reverse osmosis membranes for biofouling mitigation. Water Res. 2014, 62, 260-270.

(20) Kochkodan, V.; Hilal, N. A comprehensive review on surface modified polymer membranes for biofouling mitigation. Desalination 2015, 356, 187-207.

(21) Rahaman, M. S.; Thérien-Aubin, H.; Ben-Sasson, M.; Ober, C. K.; Nielsen, M.; Elimelech, M. Control of biofouling on reverse osmosis polyamide membranes modified with biocidal nanoparticles and antifouling polymer brushes. J. Mater. Chem. B 2014, 2, 1724.

(22) Zhang, S.; Qiu, G.; Ting, Y. P.; Chung, T. S. Silver-PEGylated dendrimer nanocomposite coating for anti-fouling thin film composite membranes for water treatment. Colloids Surfaces A Physicochem. Eng. Asp. 2013, 436, 207-214.

(23) Ye, G.; Lee, J.; Perreault, F.; Elimelech, M. Controlled Architecture of Dual-functional Block Copolymer Brushes on Thin-Film Composite Membranes for Integrated "Defending" and "Attacking" Strategies against Biofouling. ACS Appl. Mater. Interfaces 2015, 7, 23069-23079.

(24) Dreyer, D. R.; Park, S.; Bielawski, C. W.; Ruoff, R. S. The chemistry of graphene oxide. Chem. Soc. Rev. 2010, 39, 228-240.

(25) Hegab, H. M.; Zou, L. Graphene oxide-assisted Membranes: Fabrication and potential Applications in desalination and water purification. J. Memb. Sci. 2015, 484, 95-106.

(26) Perreault, F.; Faria, A. F. De; Elimelech, M.; Fonseca de Faria, A.; Elimelech, M.; Faria, A. F. De; Elimelech, M. Environmental applications of graphene-based nanomaterials. Chem. Soc. Rev. 2015, 44, 5861-5896.

(27) Zinadini, S.; Zinatizadeh, A. A.; Rahimi, M.; Vatanpour, V.; Zangeneh, H. Preparation of a novel antifouling mixed matrix PES membrane by embedding graphene oxide nanoplates. J. Memb. Sci. 2014, 453, 292-301. 
(28) Choi, W.; Choi, J.; Bang, J.; Lee, J.-H. Layer-by-layer assembly of graphene oxide nanosheets on polyamide membranes for durable reverse-osmosis applications. ACS Appl. Mater. Interfaces 2013, 5, 12510-12519.

(29) Akhavan, O.; Ghaderi, E. Toxicity of graphene and graphene oxide nanowalls against bacteria. ACS Nano 2010, 4, 5731-5736.

(30) Musico, Y. L. F.; Santos, C. M.; Dalida, M. L. P.; Rodrigues, D. F. Surface Modification of Membrane Filters Using Graphene and Graphene Oxide-Based Nanomaterials for Bacterial Inactivation and Removal. ACS Sustain. Chem. Eng. 2014, 2, 1559-1565.

(31) Sanchez, V. C.; Jachak, A.; Hurt, R. H.; Kane, A. B. Biological interactions of graphenefamily nanomaterials: an interdisciplinary review. Chem. Res. Toxicol. 2012, 25, 15-34.

(32) Perreault, F.; Tousley, M. E.; Elimelech, M. Thin-Film Composite Polyamide Membranes Functionalized with Biocidal Graphene Oxide Nanosheets. Environ. Sci. Technol. Lett. 2014, No. 1, 71-76.

(33) Marcano, D. C.; Kosynkin, D. V; Berlin, J. M.; Sinitskii, A.; Sun, Z.; Slesarev, A.; Alemany, L. B.; Lu, W.; Tour, J. M. Improved synthesis of graphene oxide. ACS Nano 2010, 4, 4806-4814.

(34) Tiraferri, A.; Yip, N. Y.; Straub, A. P.; Romero-Vargas Castrillon, S.; Elimelech, M. A method for the simultaneous determination of transport and structural parameters of forward osmosis membranes. J. Memb. Sci. 2013, 444, 523-538.

(35) Li, Q.; Elimelech, M. Organic Fouling and Chemical Cleaning of Nanofiltration Membranes: Measurements and Mechanisms. Environ. Sci. Technol. 2004, 38, 46834693.

(36) Glueckstern, P.; Priel, M.; Gelman, E.; Perlov, N. Wastewater desalination in Israel. Desalination 2008, 222, 151-164.

(37) Bar-Zeev, E.; Zodrow, K. R.; Kwan, S. E.; Elimelech, M. The importance of microscopic characterization of membrane biofilms in an unconfined environment. Desalination 2014, $348,8-15$.

(38) Tuinstra, F.; Koenig, L. Raman Spectrum of Graphite. J. Chem. Phys. 1970, 53, 11261130.

(39) Mcallister, M. J.; Li, J.; Adamson, D. H.; Schniepp, H. C.; Abdala, A. A.; Liu, J.; Herreraalonso, M.; Milius, D. L.; Car, R.; Prud'homme, R. K.; et al. Single Sheet Functionalized Graphene by Oxidation and Thermal Expansion of Graphite. Chem. Mater. 2007, 19, 4396-4404.

(40) Chen, J.; Peng, H.; Wang, X.; Shao, F.; Yuan, Z.; Han, H. Graphene oxide exhibits broadspectrum antimicrobial activity against bacterial phytopathogens and fungal conidia by intertwining and membrane perturbation. Nanoscale 2014, 6, 1879-1889.

(41) Castrillón, S. R.-V.; Perreault, F.; de Faria, A. F.; Elimelech, M. Interaction of Graphene Oxide with Bacterial Cell Membranes: Insights from Force Spectroscopy. Environ. Sci. Technol. Lett. 2015, 2, 112-117. 
(42) Li, Y.; Yuan, H.; von dem Bussche, A.; Creighton, M.; Hurt, R. H.; Kane, A. B.; Gao, H. Graphene microsheets enter cells through spontaneous membrane penetration at edge asperities and corner sites. Proc. Natl. Acad. Sci. U. S. A. 2013, 110, 12295-12300.

(43) Ruiz, O. N.; Fernando, K. A S.; Wang, B.; Brown, N. A; Luo, P. G.; McNamara, N. D.; Vangsness, M.; Sun, Y.-P.; Bunker, C. E. Graphene oxide: a nonspecific enhancer of cellular growth. ACS Nano 2011, 5, 8100-8107.

(44) Dreyer, D. R.; Todd, A. D.; Bielawski, C. W. Harnessing the chemistry of graphene oxide. Chem. Soc. Rev. 2014, 43, 5288.

(45) Mangadlao, J. D.; Santos, C. M.; Felipe, M. J. L.; Leon, A. C. C. De; Rodrigues, D. F. On the antibacterial mechanism of graphene oxide (GO) Langmuir - Blodgett films. Chem. Commun. 2015, 1, 1-4.

(46) Perreault, F.; de Faria, A. F.; Nejati, S.; Elimelech, M. Antimicrobial Properties of Graphene Oxide Nanosheets : Why Size Matters. ACS Nano 2015, 9, 7226-7236.

(47) Shilton, S. J.; Prokhorov, K. A; Gordeyev, S. A; Nikolaeva, G. Y.; Dunkin, I. R.; Smith, W. E.; Pashinin, P. P. Raman spectroscopic evaluation of molecular orientation in polysulfone. Laser Phys. Lett. 2004, 1, 336-339.

(48) Kim, H. J.; Fouda, A. E.; Jonasson, K. In Situ Study on Kinetic Behavior during Asymmetric Membrane Formation via Phase Inversion Process Using Raman Spectroscopy. J. Appl. Polym. Sci. 1999, 75, 135-141.

(49) Morra, M. On the molecular basis of fouling resistance. J. Biomater. Sci. Polym. Ed. 2000, $11,547-569$.

(50) Shaffer, D. L.; Jaramillo, H.; Romero-Vargas Castrillón, S.; Lu, X.; Elimelech, M. Postfabrication modification of forward osmosis Membranes with a poly(ethylene glycol) block copolymer for improved organic fouling resistance. J. Memb. Sci. 2015, 490, 209219.

(51) Ang, W. S.; Elimelech, M. Protein (BSA) fouling of reverse osmosis membranes: Implications for wastewater reclamation. J. Memb. Sci. 2007, 296, 83-92.

(52) Mo, Y.; Xiao, K.; Shen, Y.; Huang, X. A new perspective on the effect of complexation between calcium and alginate on fouling during nanofiltration. Sep. Purif. Technol. 2011, $82,121-127$.

(53) Jin, F.; Lv, W.; Zhang, C.; Li, Z.; Su, R.; Qi, W.; Yang, Q.-H.; He, Z. High-performance ultrafiltration membranes based on polyethersulfone-graphene oxide composites. RSC Adv. 2013, 3, 21394.

(54) Zhao, C.; Xu, X.; Chen, J.; Yang, F. Optimization of preparation conditions of poly(vinylidene fluoride)/graphene oxide microfiltration membranes by the Taguchi experimental design. Desalination 2014, 334, 17-22.

(55) Soroush, A.; Ma, W.; Silvino, Y.; Rahaman, M. S. Surface modification of thin film composite forward osmosis membrane by silver-decorated graphene-oxide nanosheets. Environ. Sci. Nano 2015, 2, 395-405. 
(56) Mejías Carpio, I. E.; Santos, C. M.; Wei, X.; Rodrigues, D. F. Toxicity of a polymergraphene oxide composite against bacterial planktonic cells, biofilms, and mammalian cells. Nanoscale 2012, 4, 4746-4756.

(57) Lee, J. J.; Chae, H.-R.; Won, Y. J.; Lee, K.; Lee, C.-H.; Lee, H. H.; Kim, I.-C.; Lee, J. J. Graphene oxide nanoplatelets composite membrane with hydrophilic and antifouling properties for wastewater treatment. J. Memb. Sci. 2013, 448, 223-230.

(58) Rafiee, J.; Rafiee, M. A.; Yu, Z. Z.; Koratkar, N. Superhydrophobic to superhydrophilic wetting control in graphene films. Adv. Mater. 2010, 22, 2151-2154. 
(a)

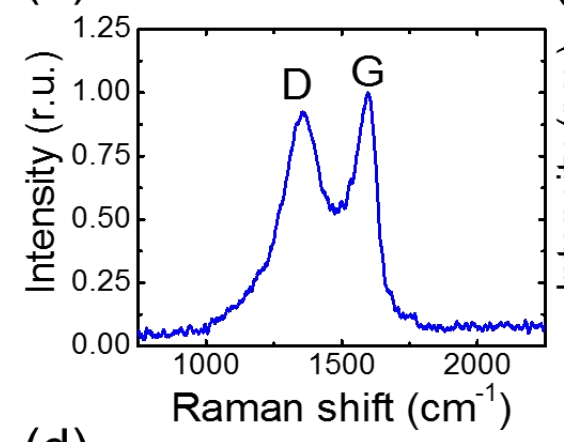

(d)

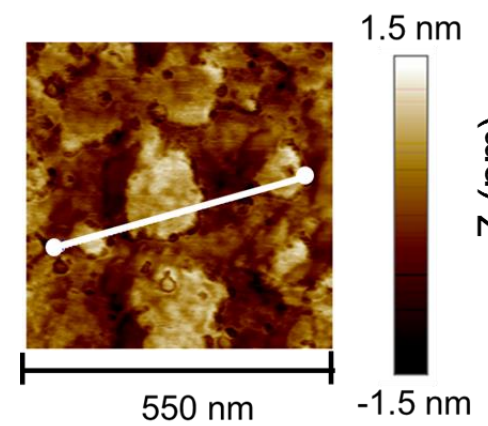

(b)

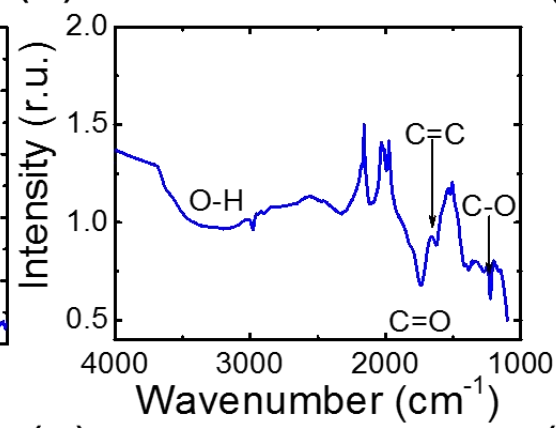

(e)

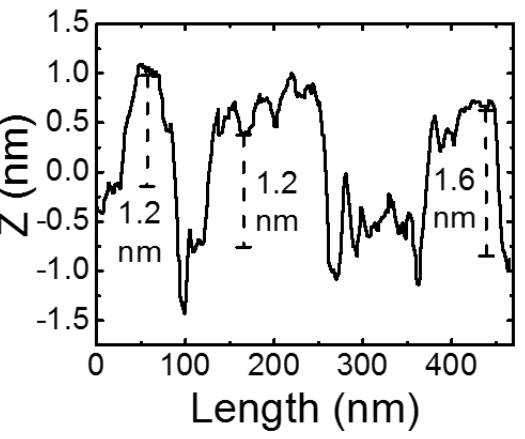

(c)

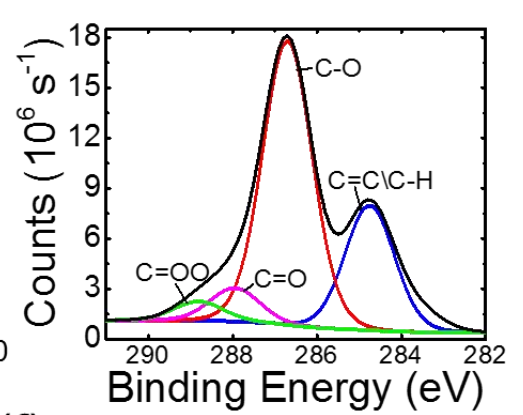

(f)

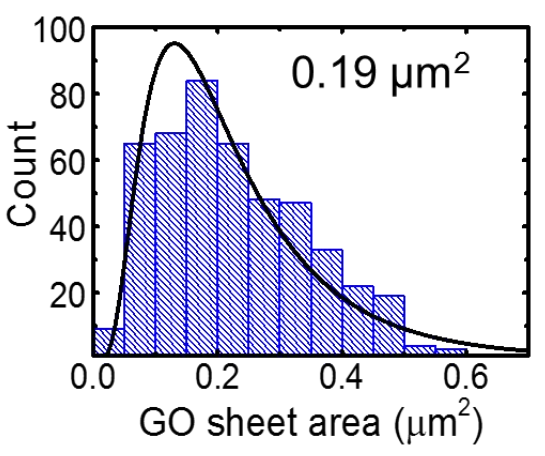

FIGURE 1. Characterization of GO nanosheets. (a) Raman spectroscopy of GO, indicating the characteristic $\mathrm{G}$ and $\mathrm{D}$ bands of carbon nanomaterials; (b) FTIR spectrum identifying the different functional groups of GO; (c) C1s XPS spectrum of GO, identifying the relative abundance of the different functional groups; (d) representative AFM image of GO sheets. The white bar indicates the thickness profile represented in (e); (e) representative sheet thickness profile obtained by AFM, indicating that GO sheets were mostly single-layer GO. (f) GO sheet area distribution, determined by image analysis of at least 2000 individual sheets obtained by SEM. 
(a)

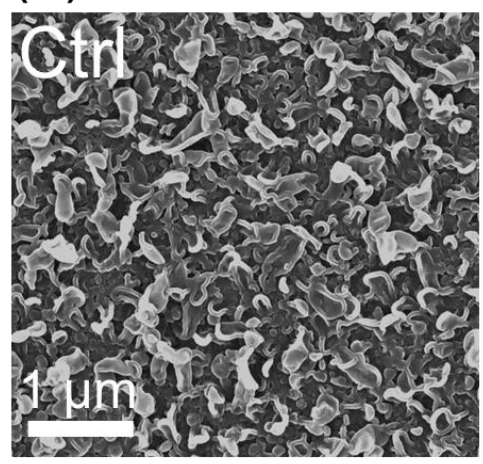

(b)

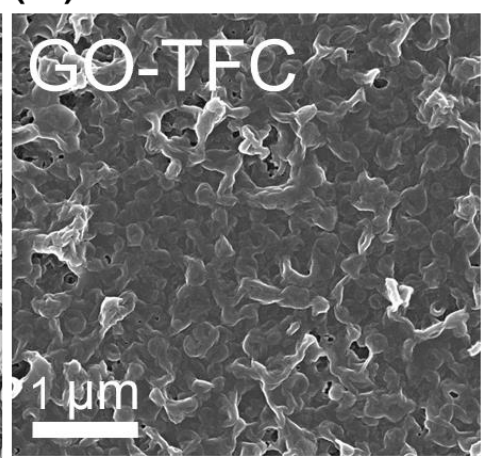

(c)

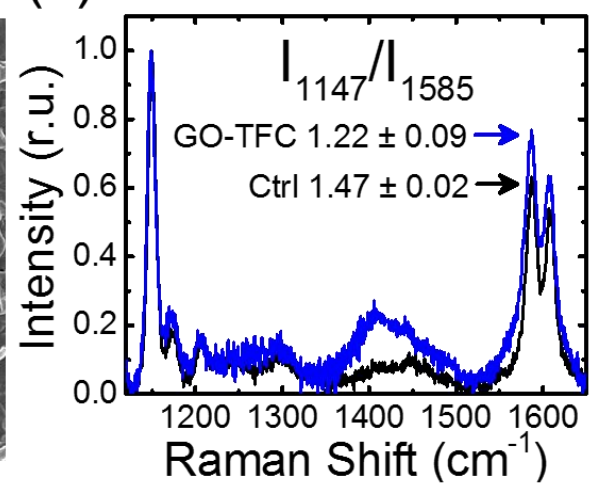

552 FIGURE 2. Characterization of TFC membranes. (a, b) Representative SEM micrographs of the

553 polyamide active layer before (a) and after (b) functionalization with GO. (c) Raman 554 spectroscopy of $\mathrm{Ctrl}$ and GO-functionalized TFC membranes. The ratio between the peaks at 5551147 and $1585 \mathrm{~cm}^{-1}$ is used as an indicator of the presence of GO on the membrane. 
(a)

(b)
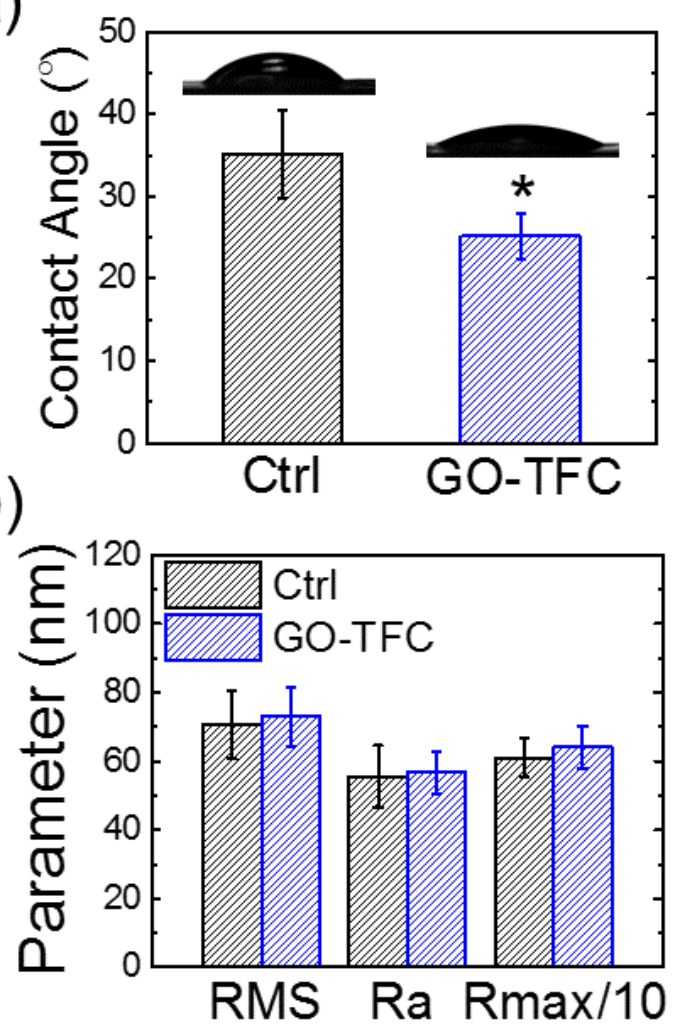

FIGURE 3. Membrane properties of pristine and GO-TFC membranes. (a) Water contact angle of Ctrl and GO-functionalized membranes. (b) Surface roughness of Ctrl and GO-functionalized membranes. RMS is the root mean-square of roughness, $R_{a}$ is the average roughness, and $R_{\max }$ is the maximum roughness. Star indicates statistical significance, determined by a student's $t$-test $566 \quad(p$-value $<0.05)$. 
(a)

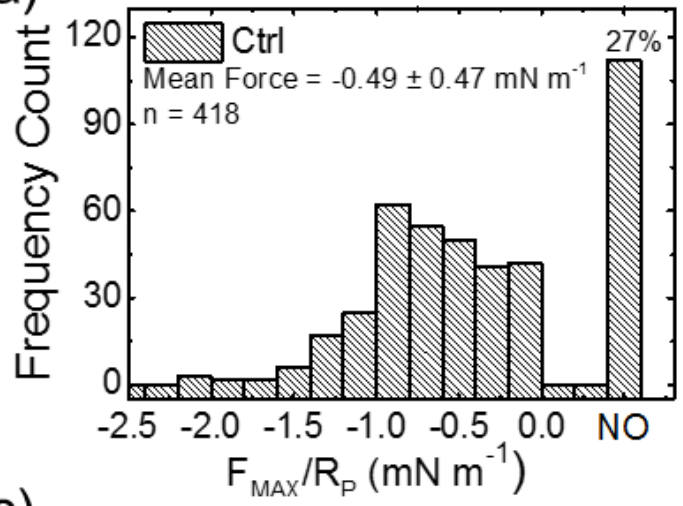

(c)

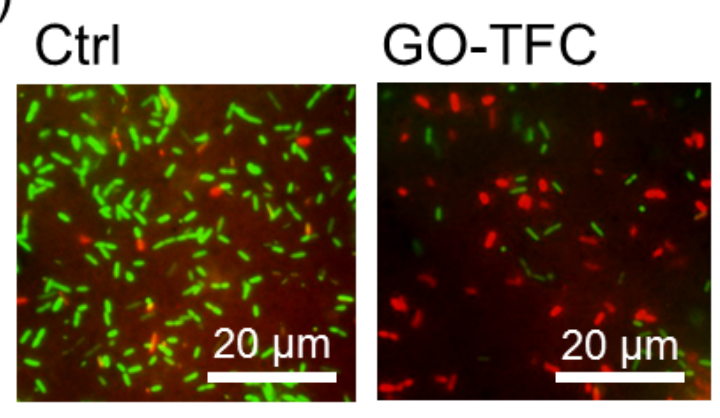

(b)

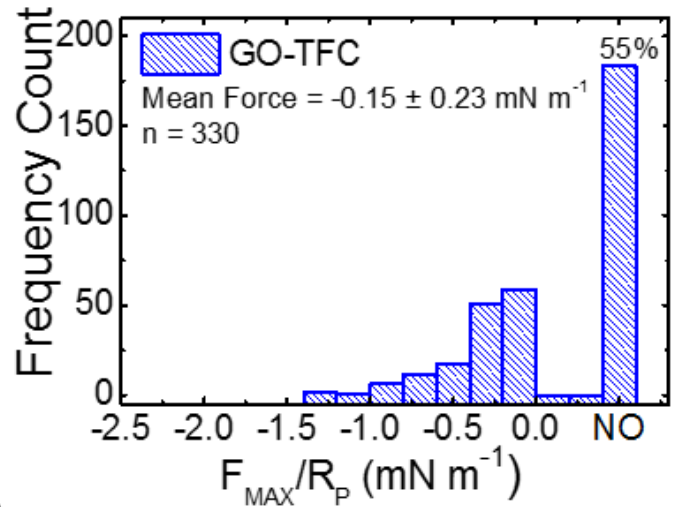

(d)

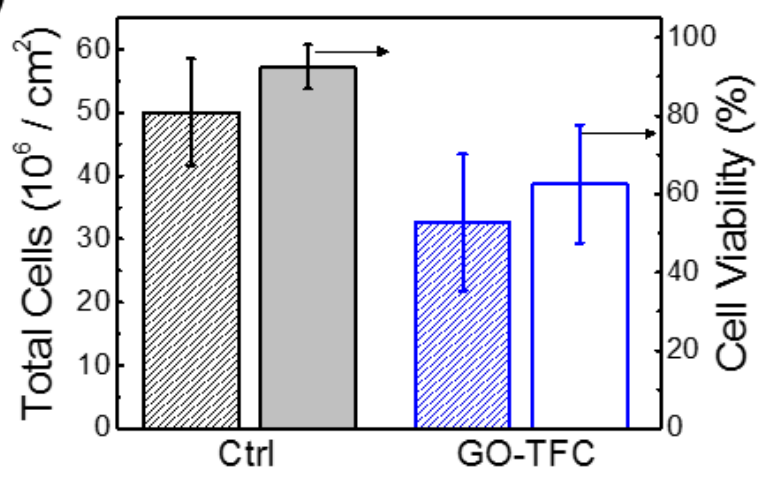

FIGURE 4. (a-b) Distribution of adhesion forces between a carboxylated latex particle probe and Ctrl (a) and GO-TFC (b) membranes. For each membrane, at least 300 force measurements, sampled over five randomly selected locations, were obtained. The columns labeled "NO" indicate measurements where the probe-membrane interactions were too weak to be differentiated from random fluctuations, and are considered as no adhesion. (c) Representative epifluorescence microscopy images of $P$. aeruginosa cells on Ctrl and GO-TFC membranes. Bacterial cells were stained with SYTO 9 (green), and PI (red) for "live" and "dead" cells, respectively. (d) Total number of $P$. aeruginosa cell adhered to the surface of Ctrl and GO-TFC membranes, and cell viability of adhered $P$. aeruginosa cells after $1 \mathrm{~h}$ of contact. Star indicates statistical significance, determined by a student's $t$-test $(p$-value $<0.05)$. 
(a)

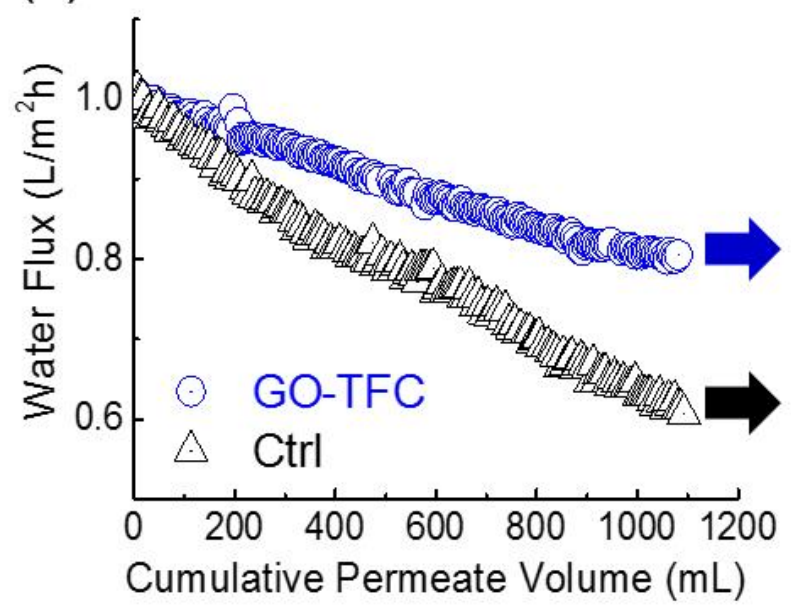

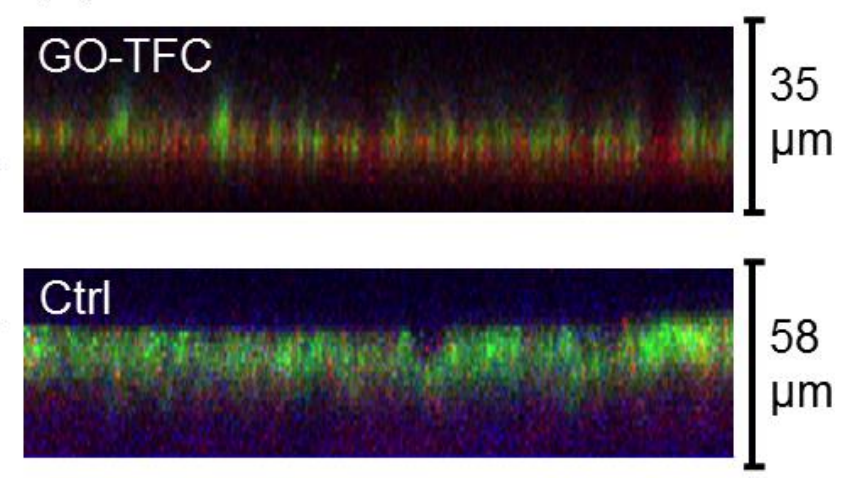

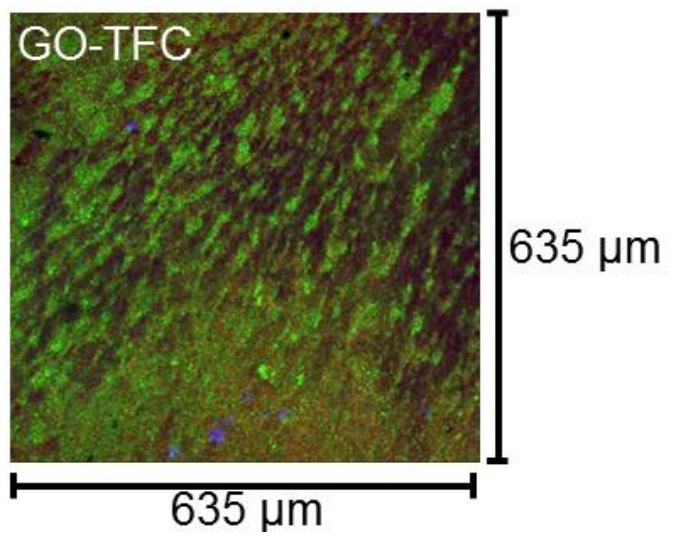

(b) (d)

\section{(c)}

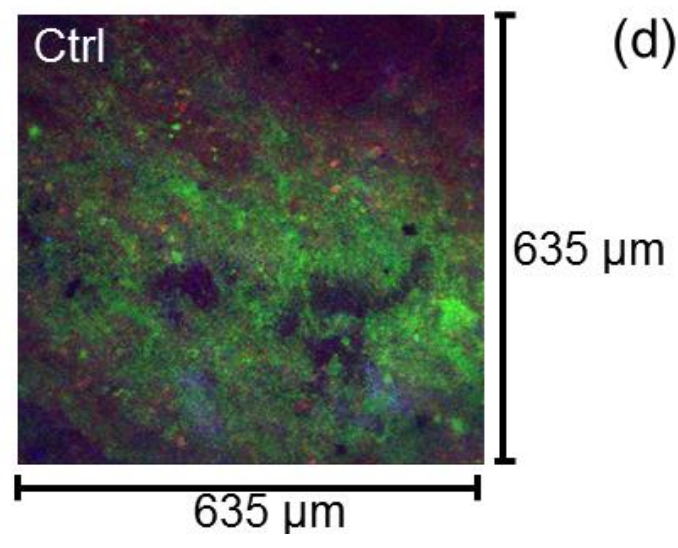

FIGURE 5. (a) Normalized water fluxes of Ctrl and GO-functionalized membrane as a function of cumulative permeate flux in biofouling experiments using $P$. aeruginosa. Feed solution was composed of synthetic wastewater matrix and $1 \mathrm{M} \mathrm{NaCl}$ was used as the draw solution. (b) Representative confocal microscopy side view of the biofilms formed on Ctrl and GO-TFC membranes after $24 \mathrm{~h}$ of FO operation. (c-d) Representative confocal microscopy top view of the biofilms formed on Ctrl (c) and GO-TFC (d) membranes after $24 \mathrm{~h}$ of FO operation. Biofilm coverage of the membrane surface is reduced by functionalization with GO. Biofilms were stained with Con A (blue), SYTO 9 (green), and PI (red) for EPS (polysaccharides), "live", and "dead" cells, respectively. 
603 TABLE 1: Biofilm characteristics of pristine and GO-functionalized membranes

604

\begin{tabular}{|c|c|c|c|c|c|c|}
\hline Parameters & $\begin{array}{c}\text { biofilm } \\
\text { thickness } \\
\mathrm{a} \\
(\mu \mathrm{m})\end{array}$ & $\begin{array}{c}\text { "live" cell } \\
\text { biovolume } \\
\mathrm{a} \\
\left(\mu \mathrm{m}^{3} / \mu \mathrm{m}^{2}\right)\end{array}$ & $\begin{array}{c}\text { "dead" } \\
\text { cell } \\
\text { biovolume } \\
\mathrm{a} \\
\left(\mu \mathrm{m}^{3} / \mu \mathrm{m}^{2}\right)\end{array}$ & $\begin{array}{c}\text { EPS } \\
\text { biovolume } \\
\mathrm{a} \\
\left(\mu \mathrm{m}^{3} / \mu \mathrm{m}^{2}\right)\end{array}$ & $\begin{array}{c}\text { TOC } \\
\text { biomass } \\
\mathrm{b} \\
\left(\mathrm{pg}^{\mathrm{u}} / \mathrm{um}^{2}\right)\end{array}$ & $\begin{array}{c}\text { total } \\
\text { protein } \\
\text { mass } \\
\left(\mathrm{pg} / \mathrm{um}^{2}\right)\end{array}$ \\
\hline $\begin{array}{c}\text { Pristine } \\
\text { membrane }\end{array}$ & $58 \pm 4$ & $15.1 \pm 2.3$ & $10.3 \pm 2.1$ & $9.8 \pm 1.5$ & $\begin{array}{c}0.47 \pm \\
0.04\end{array}$ & $\begin{array}{c}49.5 \pm \\
5.1\end{array}$ \\
\hline $\begin{array}{c}\mathrm{GO} \\
\text { modified } \\
\text { membrane }\end{array}$ & $35 \pm 6$ & $10.2 \pm 3.4$ & $14.5 \pm 2.8$ & $7.9 \pm 2.4$ & $\begin{array}{c}0.18 \pm \\
0.11\end{array}$ & $\begin{array}{c}23.9 \pm \\
3.5\end{array}$ \\
\hline
\end{tabular}

$605{ }^{a}$ biofilm thickness and biovolume were averaged, with standard deviation (SD) calculated from 606 ten random samples in duplication experiments. ${ }^{\mathrm{b}}$ Average TOC and protein biomasses were 607 presented with SD calculated from four measurements of two membrane coupons. 